\title{
Una aproximación a las funciones, motivaciones y percepciones en el cuidado familiar en contextos de cáncer en el Perú ${ }^{1}$
}

\author{
Carlos Chirinos \\ DAFITS, Universitat Rovira i Virgili \\ carlosalonso.chirinos@gmail.com
}

Resumen: El presente artículo explora algunas dimensiones del cuidado familiar en contextos de enfermedad, específicamente en situaciones de cáncer de cuello uterino en el Perú. Se analizan tres dimensiones del cuidado familiar: una primera relacionada con las funciones del cuidado, las tareas que desempeñar y el tipo de cuidados que supone; una segunda relacionada con las motivaciones que impulsan a las mujeres o demás miembros de la familia a hacerse cargo de los cuidados principales; y una tercera relacionada con los significados de un cuidado correcto, un ideal de cuidado. Si bien la expresión cuidado familiar nos invita a pensar en un cuidado compartido por cada uno de los miembros que la componen, este se presenta engañoso e invisibiliza todo el trabajo que desempeñan unas pocas protagonistas que se ven desbordadas a medida que avanza el proceso de la enfermedad. El deber moral y social adjudicado a las mujeres como cuidadoras las obliga a no descuidar ninguno de los campos donde participan - la familia, el trabajo y la enfermedad-, lo que conlleva una sobrecarga de responsabilidad, algún descuido ineludible y, finalmente, una serie de dilemas que cuestionan su papel como cuidadoras y mujeres.

Palabras clave: cáncer; cáncer de cuello uterino; cuidado familiar; dependencia; Perú.

1 El presente artículo se basa en una investigación realizada durante el año 2008 en Perú como parte de los estudios de formación para la obtención del grado de Licenciatura en Antropología (<http://tesis.pucp.edu. pe/repositorio/handle/123456789/6384>). En este documento se muestran algunos temas que formaron parte central de la tesis. 
Abstract: This article explores some dimensions of family care in the context of disease, specifically in cases of cervical cancer in Peru. Three dimensions of family care are analyzed, a first related to the functions of care, the tasks to be performed and the type of care involved. A second related to the motivations that drive women or other family members to take over the main care. And a third related to the meaning of a «proper care», an ideal care. While the term «family care» invites us to think of a shared care by all members of the family, it is presented misleading, obscuring the work they perform a few players. The moral and social duty awarded to women as caregivers forced to not neglect any of the fields where involved: family, work and disease, bringing an overload of responsibility, an inevitable neglect and finally a series of dilemmas that challenge their role as caregivers and women.

Keywords: cancer; cervical cancer; family care; dependency; Perú.

\section{Introducción}

Desde que Sontag (2003) definiera el cáncer durante la década de los años setenta del siglo pasado como una enfermedad arraigada en el lenguaje de la sociedad, podemos decir que su mención no ha caído en desuso. Más bien se ha incrementado, y se ha forjado cada vez más un espacio en la sociedad difícil de desarraigar, hasta tal punto que se la puede llegar a considerar como una de las enfermedades más conocidas en el mundo, no solo por lo recurrente que resulta en el imaginario colectivo, sino principalmente por los datos epidemiológicos que se tienen de ella. Según la Organización Mundial de la Salud, el cáncer fue durante el año 2012 la principal causa de muerte a nivel mundial (OMS, 2015). En el Perú, para situarnos, esta misma enfermedad fue durante el año 2013 la primera causa de muerte (MINSA, 2014), y el cáncer de cuello uterino fue el mayor causante de las defunciones (14\%); seguidamente figura el cáncer de mama (11\%), así como otros tipos de cánceres que terminan elevando la tasa de mortalidad en mujeres al 61 \% (INEN, 2014). Así, podemos afirmar que, para el caso peruano, el cáncer es un problema principalmente femenino.

Centrándonos en el cáncer de cuello uterino, podemos señalar que esta situación se agrava cuando conocemos la alta asociación que existe entre esta enfermedad y la pobreza. Cabe decir que, cuando hablamos de cáncer de cuello uterino en el Perú, hablamos de mujeres asociadas a viviendas y condiciones 
laborales precarias, así como a un acceso restringido a la salud, la educación y al agua potable (Benites, Rodríguez, Mezones, 2013; Gutiérrez, Alarcón, 2008). Hablamos de un problema de salud pública que abarca dos dimensiones principales: la del género y la clase social, condiciones sociales estrechamente vinculadas con una incidencia de la enfermedad que repercute en las historias de vida de las mujeres y en su acceso a la salud. $\mathrm{O}$ lo que es lo mismo, un tipo de violencia estructural que modifica y afecta a un trato igualitario en la salud y la vida (Farmer, Nizeye, Keshavjee, 2006). De esta manera, el racismo, la inequidad de género o la pobreza, tanto como las políticas públicas que las refuerzan, no serían más que las fuerzas sociales que determinan, en una gran variedad de casos, quién cae enfermo y quién tiene acceso a la sanidad.

Por otro lado, los impactos que este tipo de cáncer generan en la mujer se traducen a escalas que trascienden el mundo celular y fisiológico. Las afectaciones que suelen testimoniarse ahondan en las representaciones sociales de la enfermedad y, sobre todo, en el cuerpo simbólico de la mujer y en las emociones que despierta, pues se trata de una enfermedad que afecta a un órgano de la mujer que, sin dejar de ser un cúmulo de tejidos, es a su vez un órgano cultural y político que representa, en gran parte, lo que se entiende por feminidad. Tratarse de cáncer de cuello uterino no solo es tratarse de una enfermedad, sino cuestionarse una serie de dimensiones culturales donde el género toma un protagonismo clave.

Del mismo modo que el mundo cultural de la mujer se ve afectado, su mundo social se ve perjudicado. Por ejemplo, las oportunidades de trabajo varían: deja de trabajar o pierde el empleo, y con ello su economía se ve trastocada. En la gran mayoría de los casos, dado el perfil de las mujeres afectadas, la presencia de la enfermedad tiende a agudizar la pobreza familiar. Si bien las principales afectadas por este tipo de cáncer son las mujeres que lo padecen, su entorno más inmediato, como es la familia, se ve igualmente afectado. El cáncer de cuello uterino es, en suma, una crisis que no solo genera alteraciones en la persona enferma y en la familia, sino que demanda, a su vez, respuestas por parte de los miembros que la componen (Baider, 2003), gran parte de ellas orientadas a la organización del cuidado de la persona enferma.

De esta manera, el cuidado familiar para los casos de cáncer de cuello uterino se constituye como un elemento esencial para afrontar la enfermedad. El cuidado familiar puede entenderse, en términos genéricos, como un conjunto de acciones 
que aportan desarrollo y bienestar a una persona dependiente durante su vida cotidiana, y que engloba un cuidado material que implica trabajo, un cuidado económico que implica un costo económico, y un cuidado emocional que implica un vínculo afectivo y sentimental (Batthyány, 2008: 178). En otro plano más amplio, el cuidado puede llegarse a entender como una categoría analítica que incluye dimensiones sociales, culturales y políticas (Hughes, 2002; Castro, Esteban, Menéndez, et alii, 2008). Sociales porque el cuidado no solo implica a la familia, sino que demanda una responsabilidad compartida tanto por el Estado como por la sociedad. Culturales porque el cuidado se trata de una construcción social, es decir, una práctica que se aprende e incorpora, y se reproduce a través de justificantes biológicos y morales ligados al género. Y políticas por la necesidad de reivindicar y desmitificar una práctica esencialista que recae únicamente en la mujer. A pesar de esta necesidad reivindicativa y de entender el cuidado como un elemento social, el cuidado es, por ahora, una práctica cotidiana socialmente invisible, depositada en la familia y específicamente en la mujer, bajo condicionantes culturales que implican responsabilidad y deber.

Remarcamos estas dimensiones del cuidado porque buscamos incidir en dos aspectos. Uno: que el cuidado en el Perú es básicamente familiar. La idea de un cuidado social está muy lejos de cobrar interés y ser problematizado en cualquier institución gubernamental e incluso en la sociedad. Y dos: el cuidado familiar no es el cuidado de toda una familia al servicio de la persona dependiente, sino de una tarea que recae principalmente en las mujeres y que conlleva múltiples tareas con gran demanda de tiempo (Esteban, 2004). No solo se trata de acciones físicas, sino de una serie de responsabilidades complejas que implican organización de tareas y de tiempo, afrontamiento de conflictos $y$ tensiones, y de emociones que no derivan necesariamente en satisfacciones y alegrías, sino también en dudas y remordimientos cuando no se puede alcanzar el ideal de cuidado.

Para la finalidad de este artículo, nuestro caso de aplicación será el cuidado familiar de mujeres enfermas de cáncer de cuello uterino tratadas en el Instituto Nacional de Enfermedades Neoplásicas de Perú, principal centro nacional especializado en el tratamiento de esta enfermedad. Explorar el campo del cuidado familiar de mujeres atendidas en esta institución pública proporciona nuevas evidencias sobre cómo sobrellevan la enfermedad las familias de escasos recursos. Desde esta perspectiva, la intención del artículo se centra en explorar 
el cuidado familiar desde una visión etnográfica, indagando en campos relacionados con la gestión, la moral y la cultura del cuidado. Para una aproximación más ordenada del problema proponemos un marco de análisis a partir de cuatro ejes temáticos: (1) respecto a las familias cuidadoras, sus características y cómo se constituyen; (2) relacionado con la organización y distribución en tareas del cuidado, lo que nos lleva a incluir una serie de distinciones con respecto a los roles, funciones y posiciones que ocupan las cuidadoras familiares; (3) respecto a las motivaciones en las responsabilidades del cuidado, a la reciprocidad y a los niveles de «sacrificio» y renuncia para la asistencia; y (4) referida a las percepciones sobre «buen cuidado familiar»y flexibilización en el tiempo.

Para el estudio en cuestión, solo se trabajó con casos correspondientes al periodo de tratamiento clínico de la enfermedad, no en estado de alta o de control, por lo que las respuestas en el cuidado tienden a reflejar dinámicas propias a esta situación. Un escenario es cuidar de una persona en los momentos iniciales de la enfermedad, en estado de choque, donde los familiares y la enferma hacen todo lo posible por comenzar el tratamiento, y otro escenario es cuidar de una persona después del tratamiento, una vez superada la neoplasia.

La información tratada en este artículo proviene de un estudio de casos, con uso de entrevistas en profundidad, observaciones y conversaciones informales en colaboración con los familiares de un grupo de pacientes con tratamiento ambulatorio durante el año $2008^{2}$. En todos los casos se trataba de familias con escasos recursos económicos y con una enfermedad en niveles intermedios o avanzados, es decir, con tratamientos que implicaban visitas continuas al hospital. Además, se buscaron casos heterogéneos, es decir, que las mujeres con cáncer provinieran tanto de la capital del país, donde se encontraba el único hospital público especializado, como de provincia. Esta característica determinó el periplo de algunas pacientes frente a otras en su acceso a un tratamiento especializado oportuno ${ }^{3}$.

\footnotetext{
2 Chirinos, Carlos (2010). Organización del cuidado familiar de pacientes con cáncer de cuello uterino durante el proceso de tratamiento de la enfermedad. Estudio de casos en el Instituto Nacional de Enfermedades Neoplásicas (INEN). Tesis de licenciatura. Lima: PUCP. <http://tesis.pucp.edu.pe/repositorio/handle/123456789/6384>.

3 Este tema de acceso a la salud y atención oncológica en el Perú no se aborda exhaustivamente en este artículo. Para mayor detalle al respecto se puede revisar la siguiente bibliografía: Chirinos, Carlos (2013): «Cáncer de cuello uterino y cuidado familiar: Una mirada a la organización del cuidado familiar en pacientes con cáncer de cuello uterino avanzado en el Instituto Regional de Enfermedades Neoplásicas, IREN Norte,
} 
A estas seis familias participantes se agradece la enorme simpatía e interés que mostraron, el recibimiento y el tiempo que dieron a mis visitas, y sobre todo su compartir desinteresado y lleno de afecto.

\section{Las familias cuidadoras}

Debemos hacer énfasis en el hecho de que, entre las características sociales del cáncer de cuello uterino, este suele presentarse en mujeres de escasos recursos económicos. Los casos estudiados no escapan de esta característica y, además, suman otra: las mujeres afectadas no solo son pobres y adultas, sino que además son madres solteras. Esta condición nos indica, por un lado, que la persona dependiente ha sido hasta momentos antes del diagnóstico un elemento activo en la economía y en el cuidado familiar, dado que trabajaba y cuidaba de sus hijos menores o nietos. Con la presencia de la enfermedad no solo empobrece más a la familia, sino que al mismo tiempo se transforma de proveedora de cuidados a dependiente de cuidados, o visto de otra manera, pasa de ser una solución a una carga familiar. Por otro lado, esta condición tripartita de madresoltera-enferma nos revela que el soporte principal provendrá principalmente de las hijas mayores que vivan tanto dentro de la unidad doméstica como fuera, y no de un posible cónyuge o pareja, enfatizando así aún más la ausencia de responsabilidad masculina en el cuidado.

Un primer escenario del cuidado familiar se centra en el tipo de familias cuidadoras, las cuales pueden estar conformadas por miembros de la familia nuclear o de la familia extensa, o bien por una combinación de ambas. De esta manera, según los miembros que compongan el entorno del cuidado podemos clasificarlas en familias cuidadoras nucleares, familias cuidadoras extensas y familias cuidadoras mixtas. En un contexto donde las enfermas de cáncer de cuello uterino son mujeres de limitados recursos económicos con escaso soporte público para el cuidado, la familia se presenta, en cualquiera de sus formas, como la principal y única alternativa de soporte.

Por peculiaridades del sistema oncológico peruano, caracterizado por la falta de acceso al tratamiento especializado y el centralismo de la atención, y por el perfil social de la mayoría de mujeres afectadas, dados sus niveles de pobreza,

zona de la Macro Región Nororiental del Perú». En: Tavares, L. (ed.), Pobreza, desigualdad y salud. Buenos Aires: CLACSO. 43-73. 
las familias cuidadoras más comunes durante estas crisis suelen ser las familias extensas y las familias mixtas.

El centralismo actual que existe en el sistema público de atención de cáncer en el Perú hace que el acceso al tratamiento sea particularmente dificultoso para mujeres de provincias (Chirinos, 2013). Cuando un grupo de ellas llega a tratarse tras una larga jornada de viajes y estancias en hospitales intermedios, gran parte de los miembros responsables del cuidado corresponden a familiares de su círculo familiar inmediato que residen en la capital del país, donde se centra la atención especializada. Valga decir, una familia cuidadora extensa con residencia en Lima. En cuanto a mujeres enfermas que viven y han hecho su vida en la capital, llega a darse la situación de que las familias cuidadoras corresponden a una combinación entre familia nuclear y extensa, es decir, a una familia cuidadora mixta (gráfico 1). En escasas oportunidades se puede afirmar que la familia nuclear se hace cargo únicamente del cuidado de la persona enferma. De esto dependería el número de mujeres adultas que aún residen en el hogar, de su experiencia en el cuidado, de la flexibilidad en sus horarios de trabajo, de sus tiempos libres y de una economía familiar algo más que favorable. Pero dado el contexto familiar de los casos estudiados con la enfermedad y dependencia de uno de sus miembros adultos, y la vulnerabilidad económica del conjunto del hogar, la unidad doméstica suele apoyarse en otras mujeres provenientes de su red de parentesco más inmediato, como suele ser la familia extensa. Para las mujeres cuidadoras, dejar de lado el trabajo es una decisión que compete muchos riesgos en una economía inestable. Se puede prescindir de él en momentos muy puntuales de la enfermedad, pero no de manera prolongada.

En gran medida, la participación activa de los miembros de la familia extensa dependerá de la cercanía en el parentesco, de las historias de vida e intensidad en la relación entre los integrantes y la persona enferma. De esta manera, los principales miembros de la familia extensa comprometidos con el cuidado suelen ser las hijas mayores que viven fuera del círculo doméstico y las hermanas. En algunos otros casos se observa también la asistencia de miembros de la familia ceremonial, como son especialmente las comadres. En cuanto a las familias nucleares, si bien no existe la colaboración por parte de los cónyuges por tratarse de parejas separadas, sí existe una participación de las hijas e hijos que aún conforman el hogar, aunque con responsabilidades y tareas distintas 
dependiendo del género y la edad. Básicamente sus roles corresponden a tareas complementarias en el cuidado y no a tareas en el cuidado principal.

Gráfico 1. Tipos de familias cuidadoras

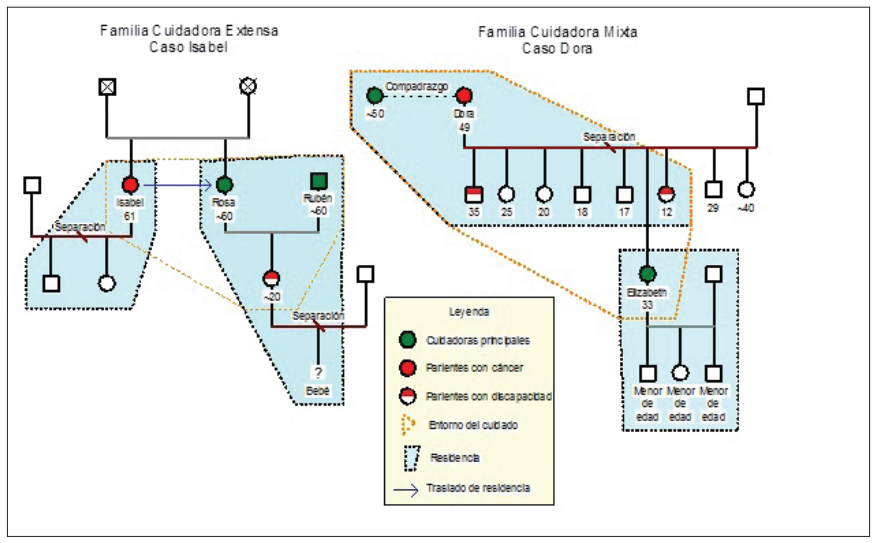

Fuente: elaboración propia

El presente gráfico trata de mostrar dos de los casos más representativos en cuanto a tipos de familias cuidadoras se refiere. Por un lado, tenemos el caso de la señora Isabel ${ }^{4}$, llegada a Lima desde Iquitos para su tratamiento; los cuidados principales recayeron en su hermana y su cuñado (tipo de familia cuidadora extensa). Por otro lado, tenemos el caso de la señora Dora, con residencia en Lima, cuyos cuidados principales estuvieron a cargo de una de sus hijas mayores, ya separada del núcleo parental, y con hogar y residencia con su propia pareja e hijos. En esta familia en especial, si bien las funciones principales las asumieron su hija mayor y su comadre (por un tiempo determinado), otras responsabilidades complementarias al cuidado provinieron de sus demás hijas e hijos menores que compartían residencia con la madre (tipo de familia cuidadora mixta). Asimismo, como nos muestra el gráfico, en ambos casos el cuidado no es una condición exclusiva hacia la persona enferma, sino que involucra en paralelo el cuidado de otros miembros del hogar que también lo exigen o requieren, ya sean bebés, niños o algún miembro con discapacidad física o cognitiva. Tanto la organización del cuidado en estos casos como la resolución de algunos dilemas en el cuidado son temas que se irán desarrollando a continuación.

4 Se usaron nombres ficticios. 


\section{Funciones en el cuidado familiar}

Si bien el cáncer, en primera instancia, se presenta como una enfermedad que genera inestabilidad y caos en las rutinas y modos de vida de las familias, con el tiempo estas tienden a reorganizarse y adaptarse como medida de afrontamiento. Entre algunas de estas medidas que se dan en el proceso, la reorganización del hogar, en cuanto a distribución de funciones y roles para el cuidado, resulta un elemento esencial.

Esta reorganización que se da como una de las primeras repuestas de adaptación a la crisis, nos permite diferenciar dos tipos de cuidados, y por ende, según corresponda, dos clases de cuidadoras. Por un lado, tenemos el cuidado primario, relacionado con tareas de asistencia permanente con la persona enferma durante gran parte del día, que incluye los lavados o baños, cambios de vestimenta, preparación y administración de los alimentos y de las medicinas, los acompañamientos y conversaciones en casa y en el hospital, y, sobre todo, las decisiones que se toman diariamente en el cuidado. Y por otro lado, tenemos el cuidado complementario, referido a actividades básicamente relacionadas con el cuidado económico de la familia, lo que implica la asistencia a los lugares de trabajo durante gran parte del día, así como el soporte eventual en tareas de cuidado, como pueden ser los acompañamientos en casa o la preparación de alimentos. Lo que distingue un tipo de cuidado de otro es el rol de gestión, de responsabilidad y de tiempos del cuidado.

Cabe señalar que asumir una responsabilidad financiera como cuidado complementario no significa en primera instancia hacerse cargo de los gastos primarios del tratamiento ${ }^{5}$, ya que estos son financiados en su mayor parte por otras fuentes, como ahorros personales, préstamos de dinero y recaudación de fondos a través de eventos como polladas ${ }^{6}$ o donaciones; significa buscar una reestabilización de la economía familiar, lo que garantiza los ingresos necesarios para la manutención del resto de los miembros de la familia y, en algunas

5 El término tratamiento está referido explícitamente a todo servicio médico usado para el control de la enfermedad, como son los exámenes clínicos (tomografías, ecografías), los análisis de laboratorio y las sesiones de radioterapia y quimioterapia.

6 Se trata de eventos populares donde se venden platos a base de pollo frito y papas asadas. La idea de estos eventos es apoyar económicamente a un fin específico, como puede ser el tratamiento de alguna enfermedad. Se trata de eventos que sirven para solucionar problemas económicos urgentes con mecanismos de reciprocidad e intercambio entre miembros de una red social (Béjar, Álvarez, 2010). 
oportunidades, cubrir algunos gastos no costosos en el cuidado en términos de alimentación y pasajes.

De esta diferenciación entre los tipos del cuidado se derivan dos tipos de cuidadoras: las cuidadoras principales y las cuidadoras complementarias. Entre ambas, el protagonismo que llegan a adoptar las cuidadoras principales se encuentra fuertemente marcado por sus grados de intervención en el cuidado, no solo por la participación directa en el trato con la persona dependiente, sino por sus labores en la gestión, delegación y coordinación de responsabilidades hacia los demás miembros de la familia, lo que refleja su importancia tanto en la categoría físico-asistencial como en el ámbito gerencial del cuidado.

A diferencia de este dualismo en las responsabilidades del cuidado principal, las cuidadoras complementarias llegan a desempeñar funciones mayormente operativas y por tiempos parciales. Muchos de estos miembros se dedican principalmente a trabajar para la reestabilización de la economía familiar o para la asistencia directa de la persona enferma durante sus tiempos libres. En este campo, el nivel de coordinaciones y gestión de funciones del cuidado tiende a ser delegado en una persona en común como es la cuidadora principal, en quien recae la mayor responsabilidad del cuidado.

Al centrarnos en las tareas directas del cuidado principal, observamos una diferenciación en las responsabilidades según el espacio donde se desempeña. De tal forma, están las tareas correspondientes a un ámbito doméstico y aquellas otras realizadas fuera del hogar o extramural. Las tareas correspondientes al cuidado doméstico se refieren a toda demanda física y emocional que se realiza dentro del hogar, como son, por ejemplo: la preparación de alimentos o dietas, la administración de medicinas, el aseo personal, además de otras exigencias físicas, como el cargar a la paciente hacia los diversos espacios de la casa - comedor, dormitorio, baño-, así como las conversaciones que se suelen tener durante gran parte del día. Por otro lado, el cuidado fuera del hogar se refiere a responsabilidades relacionadas con un ambiente público-hospitalario, como la tramitación de documentos, los turnos para los diferentes servicios, la realización de pagos de exámenes, análisis y medicinas, acudir a las citas médicas o asistir a reuniones con las asistentas sociales. Si bien estas tareas pueden sonar como responsabilidades puntuales que realizar, el acompañar a una paciente al hospital denota, además de tiempo, un sentido emocional que 
se refleja en las conversaciones que se suelen dar entre la enferma y la cuidadora entre los pasillos del hospital.

En la gran mayoría de los casos estudiados, el cuidado principal ha recaído en un solo miembro de la familia, mientras que, en otros pocos casos, esta responsabilidad ha sido más bien compartida a lo sumo por dos miembros de la familia. La elección del miembro que se dedicará al cuidado principal dependerá básicamente del género, la edad y la experiencia previa en cuidados. Es decir, una persona será más «apta» para el cuidado: uno, si es mujer; dos, si entre las mujeres de la familia esta es la de mayor edad; y tres, si dentro de este grupo de mujeres esta es la que reúne más experiencia en el cuidado. Quedan excluidos, en general, menores de edad y hombres, además de miembros con alguna discapacidad física o cognitiva. Es poco recurrente que el cuidado principal recaiga en un hombre o que sea compartido con este. El caso de Isabel representa la excepción (véase gráfico 1). Sin embargo, hay que señalar que Rubén, el cuñado, compartía responsabilidades principales porque no había otra opción. En casa, tenían otras dos personas más que cuidar: un bebé y una hija con discapacidad, de quienes se encargaba Rosa, la esposa de Rubén y hermana de Isabel. Además, no había más mujeres o miembros en los que se pudiera delegar este cuidado. Únicamente se trataban de dos personas «aptas» compartiendo la misma residencia: Rosa y Rubén.

Siguiendo con la descripción del cuidado, cuando las tareas principales no se centralizan en una sola persona, sino que son compartidas por dos miembros, se llega a observar claramente una diferenciación en las funciones según los espacios del cuidado. De esta manera, las cuidadoras que se dedican al cuidado doméstico son aquellas que, por responsabilidades en el hogar, como es el cuidado de sus propios hijos o pareja, se quedan en casa realizando una doble función del cuidado: una destinada a su propia familia y otra destinada a la enferma de cáncer. Por otro lado, las cuidadoras que se dedicaban al cuidado fuera de casa son aquellas que, por lo manejable de su situación como «amas de casa» (con esposos que las apoyaban con las tareas domésticas) o como trabajadoras (por tener negocios autónomos), podían organizarse y darse tiempo libre para asumir responsabilidades del cuidado fuera del hogar durante el tratamiento de la enfermedad. Este es el caso de Justina, con dos hijas mayores, ambas cuidadoras principales que, según sus propios contextos domésticos y laborales, 
dispusieron encargarse cada una por separado de las tareas de cuidado dentro de casa y de las tareas de cuidado en el centro hospitalario (gráfico 2).

Queda claro que las cuidadoras principales son el eje del cuidado dado el tiempo dedicado a las funciones asistenciales, pero también por su responsabilidad en la organización del cuidado. Si bien este rol las lleva en ocasiones a designar tareas de cuidado complementarias sobre los demás miembros de la familia, distribuyendo ciertos roles y tiempos para desempeñar, también existen espacios en común donde los acuerdos se toman en el conjunto familiar. En estos casos la opinión de cada miembro tiende a ser escuchada y debatida, sobre todo por los de mayor edad. Tales situaciones ocurren cuando existe la necesidad de reunir fondos para el tratamiento (a través de préstamos, empeños o polladas) o cuando se busca hacer frente al decaimiento emocional de la persona dependiente. En este último caso, el acuerdo al que llegan las cuidadoras principales, las cuidadoras complementarias y demás miembros de la familia es no contribuir a la «melancolía», ya sea llorando o mostrando cualquier otro gesto o acción que evidencie tristeza o «debilidad».

Sin embargo, así como se llegan a consensos en común y a cierta coordinación en la delegación de responsabilidades complementarias, también se registran situaciones de tensión o conflicto, originadas principalmente por descuidos o «negligencias». En estos casos, los descuidos provienen de las propias cuidadoras principales, que llegan a ser cuestionadas por las mismas enfermas o por los demás miembros de la familia. Debemos pensar que por cada responsabilidad que adquieren las cuidadoras principales, se da, en paralelo, el descuido de otra responsabilidad asumida previamente en su vida laboral o familiar. Esto sucede, por ejemplo, con aquellas cuidadoras que velan al mismo tiempo por sus propias familias (hijos, parejas), y que mantienen en una balanza dilemas morales sobre a quiénes cuidar y a quiénes descuidar durante el proceso de la enfermedad. Asimismo, en contextos donde el factor económico es apremiante, las responsabilidades laborales desatendidas a raíz de la crisis tienden a ser reconsideradas después de un tiempo por las cuidadoras principales en perjuicio del cuidado de la enferma. Esto nos indica que los cuidados principales en estos contextos se asientan sobre una base muy delicada, haciendo del cuidado un contínuum irregular con vaivenes que dependen de las propias coyunturas y responsabilidades familiares y laborales, y recayendo sobre unas pocas cuidadoras la decisión de elegir a quién se deja de lado y a quién se cuida. Un ejemplo es el caso de 
Justina, que con dos cuidadoras principales — sus hijas Julia y Claudia - que se distribuían el cuidado dentro y fuera del hogar, a lo largo de la trayectoria de la enfermedad fueron mostrando ciertas irregularidades. Julia, al ver que su madre mostraba cierta mejoría, dejó de acompañarla regularmente a sus sesiones de radioterapia. En vez de ello, centró el cuidado en sus hijos menores, así como en su negocio de tendera en un mercado de la ciudad. Por su parte, Claudia se mostraba cada vez más impuntual en la preparación de los alimentos. Ella señalaba que su tardanza se debía a otros deberes que le demandaban tiempo por la mañana, como recoger a sus niños de la escuela y prepararles el alimento, además de cuidar de su hija de dos años. Ambas cuidadoras intentaban día a día poder repartirse un cuidado que las desbordaba, lo que generaba, como consecuencia, cuidados irregulares en cada uno de los espacios donde intervenían: laboral, familiar y enfermedad. Esta situación condujo finalmente a tensiones entre las cuidadoras, entre estas y la madre, y entre las cuidadoras y el resto de miembros, y sobre todo a conjeturas y dilemas de las propias cuidadoras que cuestionaban su rol como portadoras «legítimas» de cuidado.

\section{Gráfico 2. El cuidado en la enfermedad y otras responsabilidades:} la familia y el trabajo

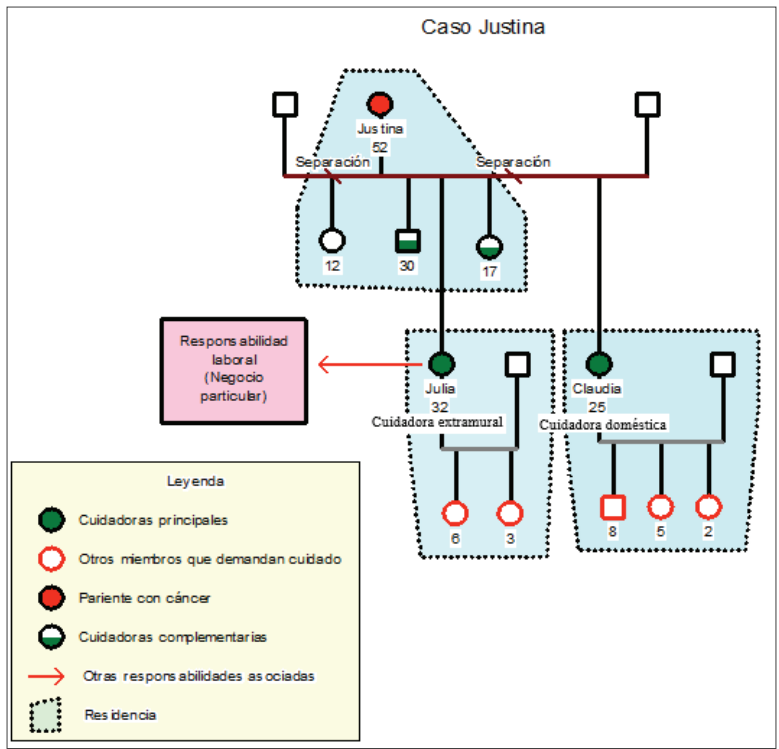

Fuente: elaboracion propia 
Es relevante seguir resaltando que las mujeres se muestran, en todos los casos estudiados, como las personas naturales en prestar cuidados tanto en su estatus de actores primarios como complementarios. Son escasas las evidencias donde los hombres se muestran como cuidadores principales. Mayormente, sus responsabilidades se concentran en funciones del cuidado complementario y no en cuestiones de gestión. Su rol es otro y depende fundamentalmente del factor laboral. Se reproduce así una tradicional división sexual del trabajo, y con ello, modelos de género que condicionan los modos de participación e implicación en los cuidados principales (Comas d'Argemir, 2015).

El tratar de entender esta lógica del cuidado como una noción femenina implica remontarse a una literatura que cuestiona este modelo, entendido como un constructo social, cultural y político (Carrasco, Borderías, Torns, 2011; West, Zimmerman, 1987). Si bien esta forma de entender el cuidado y cuestionar las lógicas y modelos que los reproducen es relevante, también es importante observar otros matices en el campo que nos permitan desmitificarlo, como suelen sugerirlo los casos donde los hombres se hacen responsables del cuidado $^{7}$, pero sin visualizarlo como una mera excepcionalidad, sino como una posibilidad de cambio en las estructuras de desigualdad y pactos sociales que distribuyen los tipos de trabajo entre los sexos.

7 Con referencia al cuidado masculino, más allá del caso de Rubén, contemplado en este artículo, resulta interesante explorar otras experiencias del cuidado masculino que han sido estudiadas en profundidad, sobre todo aquellas realizadas durante la ancianidad. Un texto que puede ayudar a introducirse en el tema es el de Milne y Hatzidimitriadou (2003) «Isn't he wonderful? Exploring the contribution and conceptualization of older husbands as carers». 


\section{Gráfico 3. Tipos de cuidado familiar}

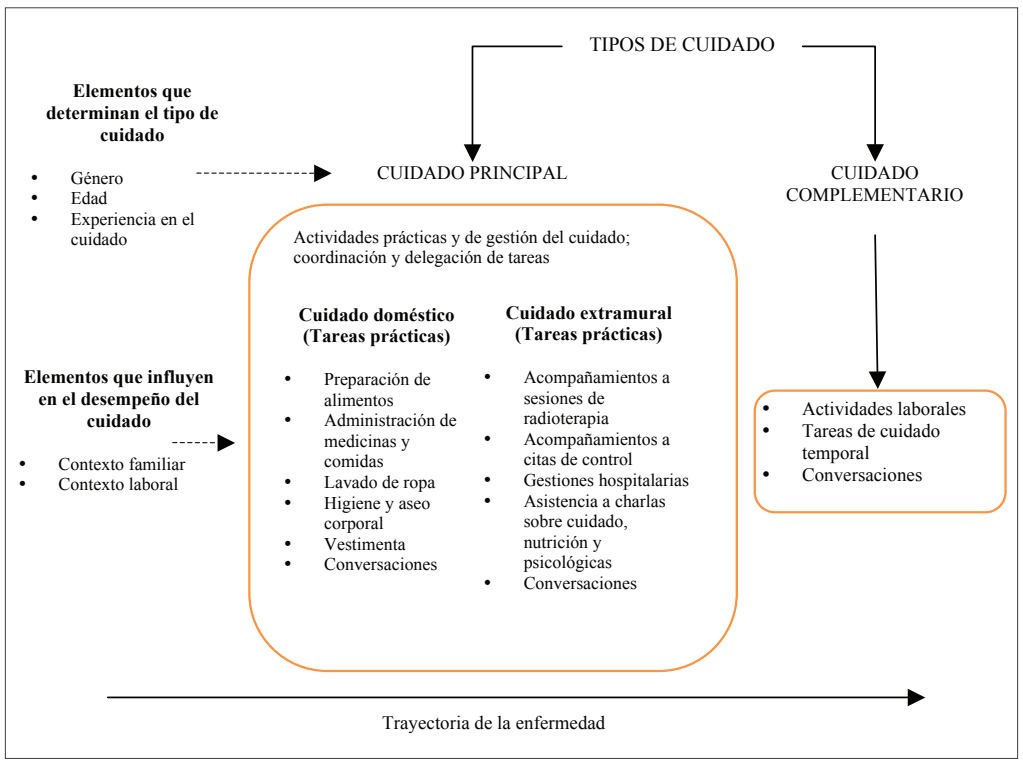

Fuente: elaboración propia

\section{Motivaciones en el cuidado familiar}

Cuanto más cercanos sean los lazos de parentesco y la intensidad en los intercambios emocionales y sociales entre un grupo de personas, mayor será la tendencia a un apoyo del grupo frente a alguna eventualidad de uno de sus miembros. Por tanto, no es casual que, en la mayoría de los casos estudiados, las principales cuidadoras sean personas relacionadas con el entorno inmediato y la vida de la persona enferma, como suelen ser las hijas, las hermanas, las comadres o los cuñados.

Una característica de este intercambio que resulta importante resaltar, y que ha significado para los casos estudiados el principio de la motivación y compromiso para el cuidado, es la retribución del afecto; un afecto brindado anteriormente por la persona dependiente hacia sus actuales cuidadoras, en función de madre, hermana, cuñada o comadre. De esta forma, las hijas revaloran la importancia que ha tenido su madre — ahora enferma - al educarlas, alimentarlas y vestirlas en contextos de mayor dificultad. Se trata de una retribución 
al trabajo realizado como madres solteras de clase trabajadora que han debido compaginar tareas del hogar y del trabajo. Por otro lado, están las hermanas cuidadoras, que retribuyen el mismo cariño y amor que anteriormente brindara la hermana enferma. El cómo se han construido las emociones, recuerdos y anécdotas de una relación entre hermanas, comadres y cuñados constituye un elemento esencial para la motivación del cuidado: desde compartir un plato de comida o un lugar donde dormir hasta brindar un consejo. La hospitalidad y la solidaridad en situaciones de necesidad o mayor apremio entre estas personas son valores que suelen retribuirse.

En esto se estaría basando el sistema de cuidado familiar en contextos de enfermedad, en un ciclo de reciprocidad donde el intercambio entre los miembros de una familia extensa, ceremonial o nuclear, hacen confluir responsabilidades, compromisos y afectos. Se trataría de un tipo de reciprocidad que no busca necesariamente una contraobligación, sino que estaría abierta a un tipo de altruismo. La estrecha distancia social hace que esta reciprocidad se mueva en una sola dirección favoreciendo al que menos tiene y más lo necesita (Sahlins, 1983). Pero aunque esta reciprocidad se trate de una ayuda más bien desinteresada, no podemos desvincularla de las obligaciones morales que se encuentran implícitas en la dadora y que empujan a realizarlas; las mismas que influyen sobre el porqué son ellas las que dan el cuidado, cómo lo dan y cómo lo sienten. En este sentido, el cuidado no solo es un mecanismo de reciprocidad generosa, sino también un deber que reproduce patrones sociales que condicionan sentimientos y obligaciones, y que lleva a las mujeres de la familia (hijas, hermanas o comadres) a asumir la responsabilidad principal del cuidado.

Del mismo modo, esta retribución del afecto ha generado en todos los casos una serie de renuncias sociales que han debido compaginar las cuidadoras principales. Asumir el cuidado significaría asumir una serie de «sacrificios» con repercusiones en la vida de la cuidadora, que van desde descuidar la propia salud, postergando visitas médicas por patologías y aflicciones; descuidar la propia familia, desatendiendo a hijos, hermanos pequeños, e incluso desvinculándose de parejas o novios; hasta descuidando la vida laboral con inasistencias a los centros de trabajo.

Cabe señalar que estas renuncias sociales no han tenido una respuesta igualitaria en todos los casos, sino que han respondido a la mayor o menor gravedad de la enfermedad. De esta forma, se puede señalar que a mayor gravedad de la 
enfermedad, mayores son las renuncias o «sacrificios», mientras que a menor gravedad las renuncias suelen ser menos frecuentes e intensas. De modo que, para los casos de mayor gravedad, las obligaciones con uno mismo, la familia y el trabajo se mantuvieron suspendidas o en segundo plano, y se prestó más atención a los cuidados de la persona dependiente. Mientras que en los casos de menor gravedad, las renuncias solo se registraron en periodos muy cortos de la crisis, y se dieron a lo largo del tratamiento un mayor número de descuidados con la enferma. Esa situación condujo a una serie de cuestionamientos y dilemas por parte de las cuidadoras, traducidos por ellas mismas como sentimientos de culpabilidad.

\section{Gráfico 4. Motivaciones para el cuidado principal}

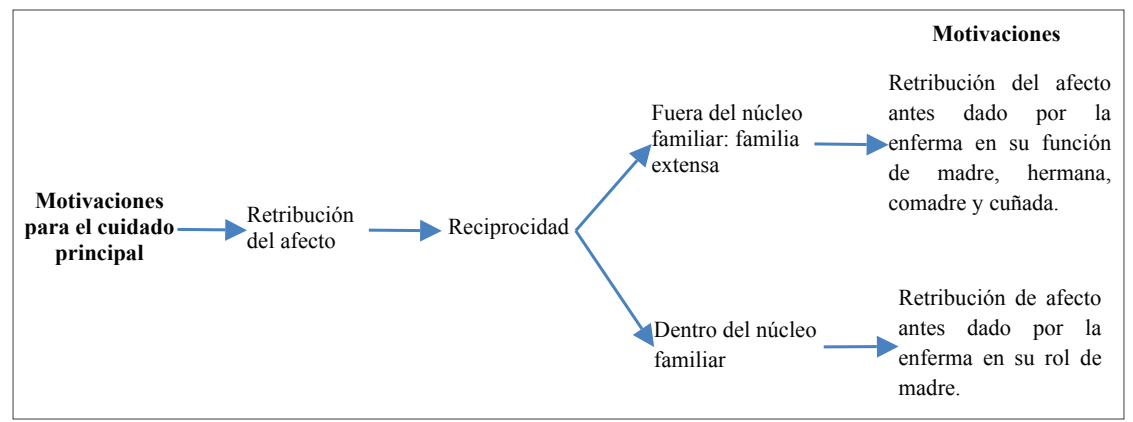

Fuente: elaboración propia

\section{Percepciones del cuidado familiar: la idea del «cuidado correcto»y su flexibilización}

El cuidado per se carece de sentido si no es entendido a partir de la propia experiencia y percepción de las protagonistas del cuidado. Para esta investigación, los significados en torno al cuidado correcto no se distinguen mucho de lo que convencionalmente en nuestra sociedad se entiende como tal. Se trata de una idea normalizada con un gran sentido moral y emocional, que, aunque muchos familiares han idealizado, en la práctica ha tendido a desdibujarse tomando otros matices. A continuación mostraremos tres aspectos que dan significado al ideal del «cuidado correcto» desde el punto de vista de las cuidadoras. 
Un primer aspecto está relacionado con el ambiente del cuidado. Esto se refiere al clima familiar al que se encuentra expuesta la persona dependiente. Dado que las familias querían transmitir sosiego y optimismo a las mujeres enfermas, el clima que se buscaba debía estar libre de situaciones caóticas o estresantes. De este modo, se evitaron discusiones o riñas entre los familiares, así como momentos de aflicción o tristeza. Otro elemento de especial cuidado resultó ser cualquier tipo de información relacionada con problemas de la economía familiar, por lo que situaciones de este tipo quedaban vetadas para conversarse abiertamente. Estaba muy claro que entre las cuidadoras y demás miembros del hogar no se podía hablar libremente de dinero, discutir ni llorar, puesto que podían afectar emocionalmente a la persona cuidada.

Este tipo de prácticas se entendían una vez que las cuidadoras explicaban su lógica. Para ellas el entorno del cuidado tenía una infinita relevancia, puesto que el estado anímico se encontraba relacionado directamente con el organismo y el cuerpo. En estos casos, encontrarse bien anímicamente era contribuir a un correcto desarrollo de los glóbulos rojos, es decir, el estar emocionalmente optimista predisponía a la persona enferma a que su cuerpo generara más hemoglobina. Que la madre, hermana o cuñada sonriera significaba que su cuerpo no se rendiría ante la enfermedad. Por el contrario, un estado de depresión producto del estrés predispondría a su cuerpo a generar menos hemoglobina $y$, con ello, una afectación más profunda de la enfermedad. La importancia que expresaban las cuidadoras por la hemoglobina se fundaba en el lenguaje médico, que señalaba que una paciente con cáncer no podía ser tratada si no conservaba los niveles de hemoglobina adecuados ${ }^{8}$. En este sentido, estar deprimida significaba tener una hemoglobina baja, y tener una hemoglobina baja significaba interrumpir el tratamiento. En un lenguaje médico-bélico, interrumpir el tratamiento significaba no atacar la enfermedad y, con ello, que la enfermedad avanzara campos que ya habían sido conquistados, y se frenaba así el camino a la recuperación.

Un segundo aspecto relacionado con el «cuidado correcto» son los acompañamientos familiares. La importancia de esta práctica en el cuidado radica en las conversaciones que se entretejen entre la persona dependiente y los familiares como resultado de esta presencia física. Diríamos que se trata de un cuidado

8 Por protocolos médicos del INEN, los tratamientos clínicos para tratar el cáncer como la radioterapia y la quimioterapia no pueden aplicarse a personas que padecen anemia. 
emocional donde la comunicación y la presencia tienen un sentido clave. Para las mujeres cuidadas, este acompañamiento les permite no sentirse solas ni olvidadas en un momento en que se cuestionan sobre el futuro, sobre la vida y la muerte. Para los familiares, se trata de una práctica que garantiza el fortalecimiento emocional de la persona cuidada para el afrontamiento de la enfermedad. Al igual que pasa con el ambiente del cuidado, el acompañamiento garantiza un buen ánimo, y por tanto, la desvinculación de preocupaciones y estrés, lo que contribuye a un mantenimiento adecuado de la hemoglobina. Además de estos acompañamientos físicos se consideran igual de importantes las visitas de amigos y otros familiares, así como las llamadas telefónicas de «seres queridos». Si bien este último tipo de comunicación no significa una presencia física, sí resulta particularmente importante para las pacientes de provincia, debido al sentimiento de proximidad que experimentaban al escuchar las voces de hijos, hijas, nietas y nietos.

Finalmente, el tercer aspecto relacionado con el «cuidado correcto» se refiere al cuidado familiar a partir de las recomendaciones médicas. Recomendaciones que, tal y como lo expresaban las cuidadoras, procuraban cumplirlas con mucha rigidez, aunque como veremos más adelante esto era muy relativo. Las recomendaciones médicas incluían cambiar las comidas cotidianas, seguir un nuevo régimen dietético dado por el hospital y administrarlo puntualmente. Asimismo, la persona enferma no podía dejar de asistir a ninguna sesión de radioterapia, quimioterapia ni consulta médica. Finalmente, los médicos sugerían que la persona enferma debía guardar reposo absoluto, traducido en cero actividades domésticas y laborales, y cero preocupaciones de cualquier tipo.

Como vemos, el «cuidado correcto» que describen las cuidadoras se trata de un cuidado referido más a un componente emocional que físico. Las descripciones sobre el cuidado físico — como vestir, trasladar, cargar o bañar-, si bien se han mencionado en los discursos, no han sido sopesadas de la misma manera que cuando se narran situaciones que implican el sentido emocional del cuidado. No hay cuestionamientos o idealizaciones que aborden tanto el tema físico, pero sí sobre cómo conversar, acompañar y actuar para transmitir alegría y entusiasmo. Los elementos más relevantes sobre el tema físico provienen principalmente de las recomendaciones médicas, como la administración de alimentos y el guardar reposo. Es curioso analizar cómo cada actor prioriza cada elemento. Por un lado, la familia adjudica más importancia al plano emo- 
tos, contraviniendo aquella idea de un ambiente libre de preocupación y estrés. Esto acarrea que la cuidadora tenga, por un lado, reclamos de los miembros de la familia exhortándola por su «dejadez», y por otro lado, de la propia enferma reclamándole por su irresponsabilidad y la falta de reciprocidad. Esta situación describe el acorralamiento emocional en el que se encuentran algunas cuidadoras. Una mezcla de sentimientos encontrados, de cansancio y de culpabilidad que ponen en cuestión su rol como mujeres. Estos sentimientos pueden entenderse como normas emocionales que reproducen patrones estandarizados y hegemónicos de lo que debe sentir un hombre y una mujer (Hochschild, 1979). De esta forma, tal como un hombre no debe llorar porque en su estatus de masculinidad radica la fuerza que lo identifica y define, la mujer debe sentirse culpable de no cumplir con un deber que la constituye en su esencia misma, la de cuidar. Esto nos indica que al margen de que la capacidad emocional humana sea universal, sentimos en unas determinadas coordenadas sociales, políticas y económicas. Se trataría de un contexto moral y político que hace que las mujeres desarrollen esa forma de vivir y sentir el cuidado (Esteban, Otxoa, 2010: 5).

Por otro lado, cabe destacar que los tiempos de mayor descuido no correspondieron a situaciones al azar, sino a periodos en los que la enferma reflejaba cierta mejoría o capacidad para realizar algunas actividades, sobre todo la de andar. A pesar de ello, se observó que la mejoría de muchas de las enfermas era inestable en el tiempo, por lo que seguía requiriendo de asistencia familiar. Que la persona dependiente pueda caminar o realizar alguna otra actividad no significaba necesariamente que se encontrara recuperada. La cronicidad de la enfermedad hace que la persona sea dependiente a lo largo de un contínuum de tiempo, aunque con intensidades distintas.

Tal como pasaba en los niveles de renuncia o «sacrificio», ser una persona enferma en estadios intermedios ${ }^{9}$ significó muchas veces que las cuidadoras principales y los familiares no dispusieran de todo su tiempo para realizar los acompañamientos domésticos u hospitalarios. Era renunciar a otras obligaciones para compartir «cara a cara» conversaciones o experiencias; una cuestión que resultaba muy valorada por la enferma en términos afectivos. Esta situa-

9 Según protocolos médicos del INEN, se consideran cuatro estadios según el grado de avance del cáncer: estadios I, II, III y iv, de los que el cuarto es el más severo. Un estadio intermedio se situaría a partir de la escala II de la enfermedad. 
ción solía darse durante gran parte del tratamiento, que iba desde el diagnóstico hasta el alta médica. Este aparente buen estado de la enferma influía en estas decisiones del cuidado. A diferencia de esta situación, las personas enfermas en estadios avanzados fueron cuidadas con una mayor rigurosidad en el tiempo, que iba desde la aparición de la enfermedad hasta mitad del tratamiento, punto en que se comenzaba a evidenciar una clara recuperación de la enferma, sobre todo en su capacidad de andar. Es preciso asentar que en ninguno de los casos el cuidado se prolongó con la misma intensidad a lo largo de la enfermedad, sino que tendió a flexibilizarse, debido, como lo señaláramos en las secciones anteriores, a contextos laborales y familiares que la cuidadora también debía cuidar y retomar, además de la aparente recuperación de la enferma, que invitaba a una desvinculación paulatina. Por mucho empeño que pusieran las mujeres en el cuidado, estas se veían desbordadas por sus diversas obligaciones en los distintos espacios donde participaban, lo que conllevaba indefectiblemente a un desmejoramiento del cuidado.

\section{Gráfico 6. Nivel de desempeño en el cuidado principal según estadios}

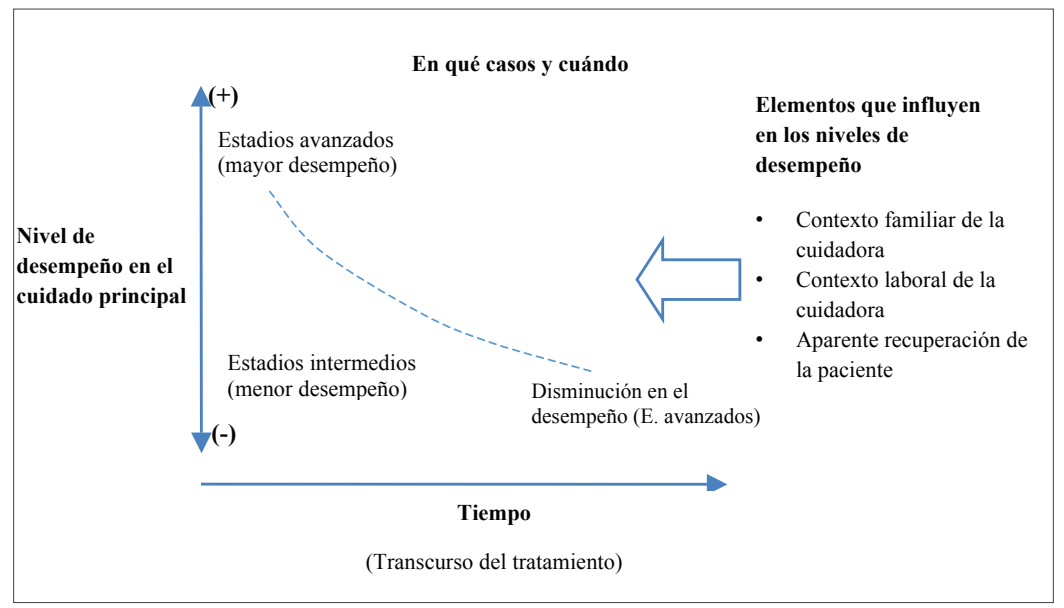

Fuente: elaboración propia 


\section{Comentario final}

Enfermar de cáncer de cuello uterino en el Perú es saber que gran parte de los cuidados recaerá en unos pocos miembros de la familia, eximiendo mayoritariamente de cualquier responsabilidad primaria a los hombres. Por otra parte, padecer esta enfermedad es saber que el cuidado no traspasará las fronteras familiares, puesto que cuidar en el Perú no es sinónimo de una responsabilidad compartida entre familia, Estado y sociedad, sino enteramente una responsabilidad familiar.

Está claro que el papel que cumple la mujer en este tipo de situaciones resulta de vital importancia para la persona enferma. En estos casos, la intensidad de las relaciones y cercanía de parentesco han resultado igualmente importantes. No en vano, las actoras primarias de este cuidado han llegado a ser las hijas, las hermanas o las comadres de la persona dependiente, quienes desde el inicio de la enfermedad contraen un compromiso significativo adjudicándose gran parte de las responsabilidades del cuidado. Queda claro también que esta responsabilidad adquirida es más bien una responsabilidad adjudicada socialmente, en que las emociones y sentimientos siguen unas directrices moldeadas cultural y políticamente. El cuidado, en este sentido, está atiborrado de una moral que empuja a las mujeres a cuidar, porque se asume como lo correcto, porque forma parte de un papel que las define como tal. Sin embargo, tal como se ha evidenciado, el cuidado no puede recaer en un solo género $y$, aún menos, en unos cuantos miembros de la familia. Debemos ser conscientes de que el cuidado demanda grandes jornadas de tiempo. Que una mujer tenga que asumir más de un tipo de cuidados en su vida diaria es criticable, como también lo es que socialmente se comparta la idea de que es en ella — la más adulta, la más experimentada — en quien debe recaer toda esa responsabilidad. Como consecuencia de esta sobrecarga, los cuidados en uno u otro espacio se han mostrado irregulares, en la vida laboral, en la vida familiar y en la vida de la enferma, lo que no hace sino menoscabar el ideal de cuidado. Por tanto, el cuidado en estos contextos no es una práctica exclusiva, sino más bien una situación que plantea dilemas sobre a quién cuidar y a quiénes descuidar, que se moldea en función de las necesidades de la persona enferma, pero también según los deberes de las mujeres cuidadoras hacia otras responsabilidades. Se trata de una situación en la que las mujeres se encuentran excedidas, agotadas y presionadas moral y socialmente. 
El poco reconocimiento que reciben refleja la poca importancia que da una sociedad a una tarea que permite que muchas mujeres $-y$ hombres - con cáncer en el Perú puedan superar la enfermedad. Se trata de un problema que debe buscar nuevas formas de redefinición cultural que contemple los poderes políticos y económicos. Retomando las palabras de Comas d’Argemir (2016: 3 ), en un contexto de hegemonía neoliberal y adelgazamiento del Estado, es especialmente importante analizar y debatir la reorganización total del trabajo desde una perspectiva holística, crítica y transformadora que incorpore la organización social de los cuidados. Se trata de entender el cuidado como un asunto social y político, y no solo como un asunto privado y de mujeres. Así, diríamos, que el cuidado no tiene por qué ser el deber de un solo género, sino un compromiso entre individuo, familia, Estado y sociedad, con el fin de contrarrestar experiencias que terminan siendo perecederas.

\section{Bibliografía}

BAIDer, L. (2003). «Cáncer y familia: aspectos teóricos y terapéuticos». Revista Internacional de Psicología Clínica y de la Salud, vol. 3, n. ${ }^{\circ}$ 1, pp. 505-520. BAt thyÁny, K. (2008). «Género, cuidados familiares y usos del tiempo». Informe final de investigación Uso del tiempo y trabajo no remunerado de las mujeres en Uruguay (2007-2008), Montevideo, UNIFEM, INE. Documento electrónico: <http://cienciassociales.edu.uy/wp-content/uploads/sites/3/2013/ archivos/Karina\%20Batthy\%C3\%A1ny\%20G\%C3\%A9nero,\%20cuidados\%20familiares\%20y\%20uso\%20del\%20tiempo.pdf> (consultado el 23 de febrero de 2016).

BéJAr, H.; Álvarez, M. (2010). «Las polladas: una estrategia de sobrevivencia en época de crisis económica y política. Lima, 1980-2003». Investigaciones Sociales, vol. 14, n* ${ }^{\circ}$ 24, pp. 259-283.

Benites, V.; Rodríguez, Y;; Mezones, E. (2013). «Determinantes sociales para cáncer de cuello uterino». Limaः INS-UNAGESP. Documento electrónico: <http://www.ins.gob.pe/repositorioaps/0/4/jer/evidencias/2013/ notas/14.Determinantes\%20sociales\%20para\%20c\%C3\%A1ncer\%20 de\%20cuello\%20uterino.pdf> (consultado el 26 de febrero de 2016). 
Carrasco, C;; Borderías, C.; Torns, T. (2011). «Introducción. El trabajo de cuidados: antecedentes históricos y debates actuales». El trabajo de cuidados. Historia, teoría y políticas. Madrid: Catarata. 13-95.

Castro, I.; Esteban, M.; Méndez, M. et. alii (2008). «No habrá igualdad sin servicios públicos y reparto del cuidado. Algunas ideas para una política feminista». En: IV Jornadas Feministas de Euskal Herria (Portugalete, 12-23 de abril). Documento electrónico: <http://www.fuhem.es/media/ecosocial/file/Boletin\%20ECOS/Boletin\%2010/PONENCIA-CUIDADOS. pdf> (consultado el 23 de febrero de 2016).

Chirinos, C. (2013). «Cáncer de cuello uterino y cuidado familiar: Una mirada a la organización del cuidado familiar en pacientes con cáncer de cuello uterino avanzado en el Instituto Regional de Enfermedades Neoplásicas, IREN Norte, zona de la Macro Región Nororiental del Perú». En: Tavares L. (ed.), Pobreza, desigualdad y salud. Buenos Aires: CLACSO. 43-73.

Comas d'Argemir, D. (2015). «Los cuidados de larga duración y el cuarto pilar del sistema de bienestar». Revista de antropología social, n. ${ }^{\circ} 24, \mathrm{pp} .375$ 404.

- (2016). «Cuidados, género y ciudad en la gestión de la vida cotidiana». En: Ramírez Kuri, P.; Valverde, C;; Meneses, M.; Suri, K. y Quiroz, H. (eds.), Ciudades. Espacios públicos en conflicto, México: Instituto de Investigaciones Sociales, UNAM.

Esteban, M. (2004). «Cuidado y salud: costes en la salud de las mujeres y beneficios sociales». En: Congreso Internacional Sare 2003, Cuidar cuesta: costes y beneficios del cuidado. Gasteiz: Emakunde-Instituto Vasco de la Mujer y Comunidad Europea/Fondo Social Europeo, pp. 63-84.

Esteban, M.; Otxoa, I. (2010). «El debate feminista en torno al concepto de cuidados». Boletín ECOS, n+ ${ }^{\circ} 10$ (ene--mar.). Documento electrónico: <http://www.fuhem.es/ecosocial/boletin-ecos/numero.aspx?n=10> (consultado el 23 de febrero de 2016).

Farmer, P; Nizeye, B.; Keshavjee, S. (2006). «Structural violence and clinical medicine». Plos Medicine, vol. 3, n. ${ }^{\circ}$ 10, pp. 1686-1691.

Gutiérrez, C; Alarcón, E. (2008). «Nivel de pobreza asociado al estadio de gravedad del cáncer ginecológico». Anales de la Facultad de Medicina, 69(4): 239-43. 
Hochschild, A. (1979) «Emotion work, feeling rules, and social structure». American Journal of Sociology, vol. 85, n.* 3 (nov.), pp. 551-575.

Hughes, C. (2002). «Care». Key concepts in feminist theory and research. London: Sage Publications. 106-129.

Instituto Nacional de Enfermedades Neoplásicas del Perú (2014). «Datos epidemiológicos. Cuadros estadísticos 2000-2013». Documento electrónico: <http://www.inen.sld.pe/portal/estadisticas/datos-epidemiologicos.html> (consultado el 3 de marzo de 2016).

Milne, A. y Hatzidimitriadou, E. (2003). «Isn't he wonderful? Exploring the contribution and conceptualization of older husbands as carers». Ageing International, vol. 28, n. ${ }^{\circ}$, pp. 389-407.

Ministerio de Salud del Perú (2014). «Principales causas de mortalidad por sexo. Perú. Año 2013». Documento electrónico: <http://www.minsa. gob.pe/estadisticas/estadisticas/Mortalidad/Macros.asp?00> (consultado el 3 de marzo de 2016).

Organización Mundial de la Salud (2015). «Cáncer. Datos y cifras». Documento electrónico: <http://www.who.int/mediacentre/factsheets/ fs297/es/ > (consultado el 3 de marzo de 2016).

Sahlins, M. (1983). «Sobre la sociología del intercambio primitivo»+ Economía de la edad de piedra. Madrid: Akal editor. 203-297.

Sontag, S. (2003). La enfermedad y sus metáforas. El sida y sus metáforas. Madrid: Suma de Letras.

West, C.; Zimmerman, D. (1987). «Doing Gender». Gender and Society, vol. 1, n. $^{\circ} 2$ (jun.), pp. 125.151 . 